\title{
Classifying Arc-Transitive Circulants
}

\author{
ISTVÁN KOVÁCS \\ ikovacs@gamma.ttk.pte.hu \\ Institute of Mathematics \& Informatics, Pécs University, 7624 Pécs, Ifjúság $u$. 6., Hungary
}

Received November 19, 2002; Revised September 4, 2003; Accepted October 27, 2003

Abstract. A circulant is a Cayley digraph over a finite cyclic group. The classification of arc-transitive circulants is shown. The result follows from earlier descriptions of Schur rings over cyclic groups.

Keywords: arc-transitive circulants, Schur rings, cyclic group

\section{Introduction}

Let $G$ be a finite group with identity element 1 . For a given set $S \subseteq G \backslash\{1\}$ define a directed graph $\Gamma$ as

$$
V \Gamma:=G, \quad E \Gamma:=\{(g, g s) \in G \times G \mid g \in G, s \in S\}
$$

In the case when $S$ is symmetric, i.e., $S=S^{-1}=\left\{g^{-1} \mid g \in S\right\}$ by $\Gamma$ we mean an undirected graph. We call $\Gamma$ a Cayley digraph over $G$ and it will be denoted by $\operatorname{Cay}(G, S)$. We will refer to $S$ as the connection set of $\Gamma$ (in [7] the term symbol is used). It follows from the definition that the left regular representation of $G$ induces a regular subgroup of Aut $\Gamma$. We will denote this subgroup as $G^{\vee}$. The full automorphism group of Cay $(G, S)$ will be also denoted by $\operatorname{Aut}(G, S)$. A circulant of order $n$ is a Cayley digraph over a cyclic group of order $n$.

Let $H$ denote a cyclic group of order $n$ and $\Gamma=\operatorname{Cay}(H, S)$. In this paper we consider arc-transitive circulants $\Gamma$, i.e., for which Aut $\Gamma$ acts transitively on the set of its arcs. If $\Gamma$ is not connected, then it can be easily seen that every component of connectedness is isomorphic to the same arc-transitive circulant of order $m$, where $m$ is a proper divisor of $n$. By this reason it will be sufficient to regard among the arc-transitive circulants the connected ones.

The desire to know all arc-transitive circulants with symmetric connection set occured in [1]. The classification then was given for those of square-free order in [7]. In this paper we classify all connected arc-transitive circulants. It should be mentioned that the complete classification was obtained by Li [6] using permutation group techniques, which method completely differs from the approach presented here. This result was appeared as reference [72] in [5]. Before to state our main results a few notions are in order.

For the (di)graphs $\Gamma$ and $\Sigma$ denote by $\Gamma[\Sigma]$ the lexicographical product of $\Gamma$ by $\Sigma$, i.e., the (di)graph with vertex set $V \Gamma \times V \Sigma$; and for $u_{1}, u_{2} \in V \Gamma$ and $v_{1}, v_{2} \in V \Sigma$ the 
pair $\left(\left(u_{1}, u_{2}\right),\left(v_{1}, v_{2}\right)\right)$ is an arc if and only if $\left(u_{1}, u_{2}\right) \in E \Gamma$ or $u_{1}=u_{2}$ and $\left(v_{1}, v_{2}\right) \in$ $E \Sigma$. If $V \Gamma=V \Sigma$, then denote by $\Gamma-\Sigma$ the (di)graph with vertex set $V \Gamma$, and arc set $E \Gamma \backslash E \Sigma$. Furthermore, $\bar{\Gamma}$ will be used for the complement of $\Gamma, m \Gamma$ will denote the (di)graph consisting of $m$ disjoint copies of $\Gamma$. $K_{n}$ will be the complete graph on $n$ vertices. The circulant $\Gamma$ defined over the cyclic group $H$ is called normal, if $H^{\vee}$ is normal in Aut $\Gamma$.

Theorem 1 Let $\Gamma$ be a connected arc-transitive circulant of order $n$. Then one of the following holds:

(a) $\Gamma=K_{n}$;

(b) $\Gamma$ is a normal circulant;

(c) $\Gamma=\Sigma\left[\bar{K}_{d}\right]$, where $n=m d$ and $\Sigma$ is a connected arc-transitive circulant of order $m$;

(d) $\Gamma=\Sigma\left[\bar{K}_{d}\right]-d \Sigma$, where $n=m d, d>3, \operatorname{gcd}(d, m)=1$ and $\Sigma$ is a connected arc-transitive circulant of order $m$.

Remark 1 In the particular case that $n$ is a square free number and $\Gamma$ is undirected Theorem 1 gives the earlier result of [7]:

Theorem 2 ([7, Theorem 1.1]) Let $\Gamma$ be a connected arc-transitive undirected circulant of order $n$. If $n$ is a square-free number and $\Gamma$ is undirected, then one of the following holds:

(a) $\Gamma=K_{n}$;

(b) $\Gamma$ is a normal circulant;

(c) $\Gamma=\Sigma\left[\bar{K}_{d}\right]$ or $\Gamma=\Sigma\left[\bar{K}_{d}\right]-d \Sigma$, where $n=m d$ and $\Sigma$ is a connected arc-transitive circulant of order $m$.

Remark 2 As it was pointed out in [7] the arc-transitive circulants in class (b) can be easily constructed. If $\Gamma=\mathrm{Cay}(H, S)$ is from class (b), then it follows (cf. [3, Lemma 2.1], [7]) that Aut $\Gamma=\left\{g \mapsto g^{\sigma} h \mid \sigma \in K, h \in H\right\}$ for a suitable group $K<$ Aut $H$, and $S=$ $\left\{g^{\sigma} \mid \sigma \in K\right\}$ for some generating element $g$ of $H$. For short we set $g^{K}=\left\{g^{\sigma} \mid \sigma \in K\right\}$.

The subgroups $K<$ Aut $H$ such that Cay $\left(H, g^{K}\right)$ is arc-transitive were described in [2]. To recall this result a few notions need to be introduced. For a given subset $T \subset H$ define $\operatorname{Stab}(T):=\{h \in H \quad \mid T h=T\}$. Let $\mathcal{P}(H)$ be the set of all prime factors of $|H|$. For $p \in \mathcal{P}(H)$ let $H_{p}$ denote the Sylow $p$-subgroup of $H$. It is well-known that $H=\prod_{p \in \mathcal{P}(H)} H_{p}$ and Aut $H=\prod_{p \in \mathcal{P}(H)}$ Aut $H_{p}$. For a given subgroup $K \leq$ Aut $H$ we write Aut $H_{p} \leq K$, if $K$ is of the form $K=\left(\right.$ Aut $\left.H_{p}\right) K^{\prime}$, where $K^{\prime} \leq \prod_{q \in \mathcal{P}(H) \backslash\{p\}}$ Aut $H_{q}$. Define

$$
\mathcal{P}^{*}(K)=\left\{p \in \mathcal{P}(H)|| H_{p} \mid=p \text {, Aut } H_{p} \leq K\right\} .
$$

Theorem 3 ([2, Theorem 6.1]) Let $H$ be a cyclic group with a generating element $g \in H$, and let $K \leq$ Aut $H$. Then Cay $\left(H, g^{K}\right)$ is normal if and only if $\left|\operatorname{Stab}\left(g^{K}\right)\right| \leq 2$ and $\mathcal{P}^{*}(K) \subset$ $\{2,3\}$.

Suppose that $H$ is a cyclic group of order $n$ and let $d$ be a proper Hall divisor of $n$, i.e., for which $\operatorname{gcd}(d, n / d)=1$. Denote by $D$ and $K$ the unique subgroups of $H$ of order $d$ and $k / d$, respectively. Then $H=D K$. If a given subset $S \subset H$ can be written of the form $S=S_{1} S_{2}=\left\{x y \mid x \in S_{1}, y \in S_{2}\right\}$ such that $S_{1} \subset D \backslash\{1\}$ and $S_{2} \subset K \backslash\{1\}$, then the 
circulant $\operatorname{Cay}(H, S)$ will be called the tensor product of Cay $\left(D, S_{1}\right)$ and $\operatorname{Cay}\left(K, S_{2}\right)$. This will be denoted by $\operatorname{Cay}(H, S)=\operatorname{Cay}\left(D, S_{1}\right) \otimes \operatorname{Cay}\left(K, S_{2}\right)$.

After further examination of the circulants $\Sigma$ occuring in classes (c) and (d) in Theorem 1 we will derive the following classification in terms of tensor products:

Theorem 4 Let $H$ be a cyclic group of order $n$, and let $g$ be a fixed generating element of $H$. Then the connected arc-transitive circulants over $H$ can be enumerated as the circulants:

$$
\left(\Gamma \otimes K_{n_{1}} \cdots \otimes K_{n_{k}}\right)\left[\bar{K}_{m}\right],
$$

where $n=n_{0} \ldots n_{k} m$ ( $k$ is possibly equal to 0$)$, the numbers $n_{i}, i \in\{0, \ldots, k\}$, are pairwise coprime and $h_{i}>3$ for all $i>0$. Furthermore, $\Gamma=K_{n_{0}}$ or $\Gamma=\operatorname{Cay}\left(\left\langle g^{n / n_{0}}\right\rangle,\left(g^{n / n_{0}}\right)^{K}\right)$ and $K \leq \operatorname{Aut}\left\langle g^{n / n_{0}}\right\rangle$ such that $\left|\operatorname{Stab}\left(\left(g^{n / n_{0}}\right)^{K}\right)\right|=1$ and $\mathcal{P}^{*}(K) \subset\{2,3\}$.

The proof of Theorem 1 and Theorem 4 will be given in Section 3. They will be obtained as a consequence of the strong structure results on Schur rings over cyclic groups proved in [4] and [2]. For the reader convenience they will be formulated in Theorems 5 and 6 in Section 2 . The definition of a Schur ring over a cyclic group, its connection with circulants, and its properties that will be needed are also presented in Section 2.

\section{Schur rings over cyclic groups}

In what follows the cyclic group of order $n$ will be presented as the additive group of the ring of residue classes modulo $n . \mathbb{Z}_{n}$ will be used to denote both this ring and its additive group. We identify $\mathbb{Z}_{n}$ with the set $\{0,1, \ldots, n-1\}$. Then $\mathbb{Z}_{n}^{\vee}$ is formed by the permutations $x \mapsto x+k, k \in \mathbb{Z}_{n}$ ( + is the additive operation of $\mathbb{Z}_{n}$ ). Let $\mathbb{Z}_{n}^{*}$ be the multiplicative group of units of $\mathbb{Z}_{n} . \mathbb{Z}_{n}^{*}$ will also denote its action over $\mathbb{Z}_{n}$ via left multiplication, $i$.e., for $k \in \mathbb{Z}_{n}^{*}$ the corresponding permutation is given by $x \mapsto k \cdot x$ ( $\cdot$ is the multiplicative operation of $\mathbb{Z}_{n}$ ).

In the basic notation of Schur rings we will follow [10]. For the cyclic group $\mathbb{Z}_{n}$, let $\mathbb{Z}\left(\mathbb{Z}_{n}\right)$ be the group ring of all formal sums $\sum_{i \in \mathbb{Z}_{n}} a_{i} i, a_{i} \in \mathbb{Z}$. It is also a $\mathbb{Z}$-module with scalar multiplication $k\left(\sum_{i \in \mathbb{Z}_{n}} a_{i} i\right):=\sum_{i \in \mathbb{Z}_{n}}\left(k a_{i}\right) i$. For a subset $T \subseteq \mathbb{Z}_{n}$ let $\underline{T}$ denote the element $\sum_{i \in T} i$ in $\mathbb{Z}\left(\mathbb{Z}_{n}\right)$. Such an element is also called a simple quantity. The transpose of $\alpha=\sum_{i \in \mathbb{Z}_{n}} a_{i} i$ is defined as $\alpha^{\top}:=\sum_{i \in \mathbb{Z}_{n}} a_{i}(-i)$.

Let $\mathcal{S}$ be a subring of $\mathbb{Z}\left(\mathbb{Z}_{n}\right)$ which as a module is generated by the simple quantities $T_{0}, \ldots, \underline{T_{r}} . \mathcal{S}$ is called a Schur ring (for short an $S$-ring) over $\mathbb{Z}_{n}$ if the sets $T_{i}$ 's form a partition of $\mathbb{Z}_{n}$ satisfying: $T_{0}=\{0\}$, and that for each $i \in\{0, \ldots, r\}$ there exists some $j \in\{0, \ldots, r\}$ such that ${\underline{T_{i}}}^{\top}=T_{j}$. The sets $T_{i}$ 's are called the basic sets of $\mathcal{S}$. The number $r+1$ is called the rank of $\mathcal{S}$. The module generated by $\underline{0}$ and $\underline{\mathbb{Z}_{n} \backslash\{0\}}$ is an S-ring, which is called the smallest $\mathrm{S}$-ring over $\mathbb{Z}_{n}$.

We remark that S-rings can be defined over any finite group $G$ by replacing $\mathbb{Z}_{n}$ with $G$ in the above definition, where for the group ring element $\alpha=\sum_{g \in G} a_{g} g \in \mathbb{Z}(G)$, its transpose is defined as $\alpha^{\top}:=\sum_{g \in G} a_{g} g^{-1}$. They were created by I. Schur to study permutation groups, cf. [10]. The idea to use S-rings over $\mathbb{Z}_{n}$ to study circulants goes back 
to the works of M. Klin and R. Pöschel. For a recent survey on this approach we refer to [9].

The automorphism group of the S-ring $\mathcal{S}$ is defined as

$$
\operatorname{Aut}(\mathcal{S}):=\bigcap_{i=1}^{r} \operatorname{Aut}\left(\mathbb{Z}_{n}, T_{i}\right),
$$

where $T_{0}=\{0\}, T_{1}, \ldots, T_{r}$ are the basic sets of $\mathcal{S}$. Clearly, $\mathbb{Z}_{n}^{\vee} \leq \operatorname{Aut}(\mathcal{S}) . \mathcal{S}$ is called normal (cf. [2]) if $\mathbb{Z}_{n}^{\vee}$ is normal in $\operatorname{Aut}(\mathcal{S})$.

For a given subset $S \subseteq \mathbb{Z}_{n} \backslash\{0\}$ there exists a least $S$-ring over $\mathbb{Z}_{n}$ containing $\underline{S}$, see [9]. Moreover, if this S-ring is denoted by $\langle\langle S\rangle\rangle$, then

$$
\operatorname{Aut}\left(\mathbb{Z}_{n}, S\right)=\operatorname{Aut}(\langle\langle S\rangle\rangle)
$$

Let $\mathcal{S}$ be an $S$-ring over $\mathbb{Z}_{n}$. We recall next a few results about the structure of the basic sets of $\mathcal{S}$. The order of the subgroup of $\mathbb{Z}_{n}$ generated by a given set $T \subseteq \mathbb{Z}_{n}$ is called the order of $T . T$ is called free if $\operatorname{Stab}(T)=\left\{i \in \mathbb{Z}_{n} \mid T+x=T\right\}$ is trivial. $\mathcal{S}$ is called free if its basic sets of maximal order are free. A subgroup $H \leq Z_{n}$ is called a $\mathcal{S}$-subgroup if $\underline{H} \in \mathcal{S}$.

Theorem 5 ([4]) Let $\mathcal{S}$ be an $S$-ring over $\mathbb{Z}_{n}$. If $\mathcal{S}$ is not free, then there exist $\mathcal{S}$-subgroups $\{0\}<H \leq K<\mathbb{Z}_{n}$ such that each basic set of $\mathcal{S}$ contained in $\mathbb{Z}_{n} \backslash K$ is a union of $H$-cosets.

Let $d$ be a Hall divisor of $n$, and assume that $D$ and $K$ are $\mathcal{S}$-subgroups of $\mathbb{Z}_{n}$ such that $|D|=d$ and $|K|=n / d$, respectively. Then $\mathbb{Z}_{n}=D+K$. Denote by $\mathcal{S}_{1}$ and $\mathcal{S}_{2}$ the S-ring over $D$ and $K$, respectively that are induced by $\mathcal{S}$. Then $\mathcal{S}$ is called the tensor product of $\mathcal{S}_{1}$ and $\mathcal{S}_{2}$, if any of its basic sets is of the form $T_{1}+T_{2}$, where $T_{1}$ and $T_{2}$ are basic sets of $\mathcal{S}_{1}$ and $\mathcal{S}_{2}$, respectively. In the particular case when $\mathcal{S}_{1}$ is of rank 2, the $S$-ring $\mathcal{S}$ is also called d-decomposable.

Theorem 6 ([2, Corollary 6.4]) A free S-ring over $\mathbb{Z}_{n}$ is not normal if and only if it is $d$-decomposable for some Hall divisor $d \mid n, d>3$.

\section{The proof of the main results}

Theorem 1 will follow from Theorems 5 and 6 using the following

Proposition 1 Let $\operatorname{Cay}\left(\mathbb{Z}_{n}, S\right)$ be a connected arc-transitive circulant. If $\{0\}<H<\mathbb{Z}_{n}$ is an $\langle\langle S\rangle\rangle$-subgroup, then $H \cap S=\emptyset$.

Proof: For short let $\mathcal{S}=\langle\langle S\rangle\rangle$. We are going to show that $S$ is a basic set of $\mathcal{S}$. Denote by $A$ the point stabilizer of $0 \in \mathbb{Z}_{n}$ in $\operatorname{Aut}(\mathcal{S})$. If $T$ is a basic set of $\mathcal{S}$, then by (1) we have that 
$T$ is fixed by $A$, hence it is the union of orbits of $A$. Since Aut $(\mathcal{S})=\operatorname{Aut}\left(\mathbb{Z}_{n}, S\right)$, see (2), and Cay $\left(\mathbb{Z}_{n}, S\right)$ is arc-transitive, $S$ is an orbit of $A$. These observations imply that $S$ is indeed a basic set of $\mathcal{S}$.

Now the proposition follows immediately. By $H$ being an $\mathcal{S}$-subgroup $H \cap S=\emptyset$ or $S \subset H$. In the latter case $\langle S\rangle=H<\mathbb{Z}_{n}$, which cannot occur because of the initial assumption that Cay $\left(\mathbb{Z}_{n}, S\right)$ is connected.

The Proof of Theorem 1: Let $\Gamma=\operatorname{Cay}\left(\mathbb{Z}_{n}, S\right)$ be a connected arc-transitive circulant and let $\mathcal{S}=\langle\langle S\rangle\rangle$. If $\Gamma=K_{n}$, then it is arc-transitive, so we may assume that $\Gamma \neq K_{n}$ and, thereby, $\mathcal{S}$ has rank more than $2 . \mathcal{S}$ is normal if and only if so is $\Gamma=\operatorname{Cay}\left(\mathbb{Z}_{n}, S\right)$, see (2), hence in what follows we can assume that $\mathcal{S}$ is not normal.

Assume that $\mathcal{S}$ is not free. Then apply Theorem 5 to conclude that there exist $\mathcal{S}$-subgroups $\{0\}<H \leq K<\mathbb{Z}_{n}$ such that for each basic set $T$ of $\mathcal{S}, T \backslash K$ is the union of some $H$-cosets. $S \cap K=\emptyset$ by Proposition 1 , and since $S$ is a basic set of $\mathcal{S}$ it follows that $S$ is the union of some $H$-cosets.

Denote by $i^{*}$ the image of $i \in \mathbb{Z}_{n}$ under the natural homomorphism of $\mathbb{Z}_{n}$ onto $\mathbb{Z}_{n / h}$, where $h=|H|$. It is easy then to check that

$$
\Gamma=\operatorname{Cay}\left(\mathbb{Z}_{n}, S\right)=\operatorname{Cay}\left(\mathbb{Z}_{n / h}, S^{*}\right)\left[\bar{K}_{h}\right]
$$

where $S^{*}=\left\{i^{*} \mid i \in S\right\}$. Cay $\left(\mathbb{Z}_{n}, S\right)$ is connected and arc-transitive if and only if so is $\operatorname{Cay}\left(\mathbb{Z}_{n / h}, S^{*}\right)$. This gives by taking $\Sigma=\operatorname{Cay}\left(\mathbb{Z}_{n / h}, S^{*}\right)$ that $\Gamma$ belongs to class (c).

It remains to consider the possibility that $\mathcal{S}$ is free. $\mathcal{S}$ is not normal is equivalent to saying that it is $d$-decomposable for some Hall divisor $d>3$, see Theorem 6 . Let $D, K<\mathbb{Z}_{n}$ with $|D|=d$ and $|K|=k=n / d$. Since $D$ and $K$ are $\mathcal{S}$-groups, we obtain as above that $S \cap D=S \cap K=\emptyset$. This implies that there exists a subset $U \subset K$ such that $S=\cup_{i \in U}((D \backslash\{0\})+i)$. From this

$$
\operatorname{Cay}\left(\mathbb{Z}_{n}, S\right)=\operatorname{Cay}\left(\mathbb{Z}_{k}, U^{\prime}\right)\left[\bar{K}_{d}\right]-d \operatorname{Cay}\left(\mathbb{Z}_{k}, U^{\prime}\right)
$$

where $U^{\prime}=\left\{i \in \mathbb{Z}_{k} \mid d i \in U\right\}$. $\Gamma$ is connected and arc-transitive if and only if so is $\operatorname{Cay}\left(\mathbb{Z}_{k}, U^{\prime}\right)$. Thus by taking $\Sigma=\operatorname{Cay}\left(\mathbb{Z}_{k}, U^{\prime}\right)$ one obtains $\Gamma$ as one of the circulants in class (d).

The Proof of Theorem 4: Let $\Gamma=\operatorname{Cay}\left(\mathbb{Z}_{n}, S\right)$ be a connected arc-transitive circulant and let $\mathcal{S}=\langle\langle S\rangle\rangle$. We are going to use the notation of the previous proof. If $\mathcal{S}$ is not free, then it follows that $H=\operatorname{Stab}(S)$ is a non-trivial $\mathcal{S}$-subgroup of $\mathbb{Z}_{n}$. From this it follows (see the above proof) that $\Gamma=\Sigma\left[\overline{K_{h}}\right]$, where $h=|H|$ and $\Sigma=\operatorname{Cay}\left(\mathbb{Z}_{n / h}, S^{*}\right)$, where $S^{*}$ is the image of $S$ under the natural homomorphism of $\mathbb{Z}_{n}$ onto $\mathbb{Z}_{n / h}$. It also follows that the S-ring $\left\langle\left\langle S^{*}\right\rangle\right\rangle$ is a free $S$-ring over $\mathbb{Z}_{n / h}$.

Therefore, it remains to describe $\Gamma$, where $\mathcal{S}$ is a free $S$-ring over $\mathbb{Z}_{n}$. If now $\Gamma \neq K_{n}$ and $\Gamma$ is not normal, then $\Gamma$ was proved to belong to class (d) in Theorem 1. More precisely, we have the connection set $S$ in the form: $S=U+(D \backslash\{0\})$, where $K$ and $U$ were suitable disjoint subgroups of $\mathbb{Z}_{n}, U \subset K$. In terms of tensor products we have $\Gamma=\Sigma \otimes K_{d}$, where 
$\Sigma=\operatorname{Cay}(K, U), d=|D|>3$ and $\operatorname{gcd}(d,|K|)=1$. It is clear that the S-ring $\langle\langle U\rangle\rangle$ over $K$ remains free. Thus the previous argument can be applied to $\Sigma$. After finitely many steps we obtain eventually $\Gamma$ as the tensor product of a circulant $\Sigma^{\prime}$ with the tensor products of a few complete graphs, where $\Sigma^{\prime}$ is complete or it is a normal circulant. Since the S-ring generated by the connection set of $\Sigma^{\prime}$ is free, the description of $\Sigma^{\prime}$ given in Theorem 4 follows from Theorem 3 .

Remark 3 We remark that in the above proof for the free S-ring $\langle\langle S\rangle$, the set $S$ could have been obtained directly from the characterization of basic sets of S-rings over cyclic groups, see [8, Theorem 3.1].

\section{Acknowledgments}

The author thanks both referees for the valuable comments and suggestions. They made many useful remarks and improvements.

\section{References}

1. B. Alspach, M.D.E. Conder, and D. Marušič, "A classification of 2-arc-transitive circulants," J. of Algebraic Combinatorics 5 (1996), 83-86.

2. S. Evdokimov and I. Ponomarenko, "Characterization of cyclotomic schemes on a finite field and normal Schur rings over a cyclic group," Algebra and Analysis 14(2) (2002), 11-55.

3. C. Godsil, "On the full automorphism group of a graph," Combinatorica 1 (1981), 243-256.

4. K.H. Leung and S.L. Ma, "The structure of Schur rings over cyclic groups," J. Pure Appl. Algebra 66 (1990), 287-302.

5. C.H. Li, "On isomorphisms of finite Cayley graphs—a survey," Discrete Mathematics 256 (2002), 301-334.

6. C.H. Li, "Permutation groups with a cyclic regular subgroup and arc-transitive circulants," submitted to J. of Combinatorics (2003).

7. C. Li, D. Marušič, and J. Morris, "Classifying arc-transitive circulants of square-free order," J. of Algebraic Combinatorics 14 (2001), 145-151.

8. M. Muzychuk, "On the structure of basic sets of Schur rings over cyclic groups," J. of Algebra 169(2) (1994), 655-678.

9. M. Muzychuk, M. Klin, and R. Pöschel, "The isomorphism problem for circulant graphs via Schur ring theory," DIMACS Series in Discrete Mathematics and Theoretical Computer Science 56 (2001), 241-264.

10. H. Wielandt, Finite Permutation Groups, Academic Press, New York, 1964. 